\title{
Testing of SARS-CoV-2 in the fight against COVID-19 pandemic
}

\section{Mekala Fernando ${ }^{1}$, Dilini Mataraarachchi ${ }^{1}$, DA Nathaniel ${ }^{2}$, Rima Niyas ${ }^{1}$, Monika Wijeratne ${ }^{3}$, Anuji Upekshika Gamage $^{4 *}$}

${ }^{1}$ Ministry of Health, Sri Lanka; ${ }^{2}$ Post Graduate Institute of Medicine, University of Colombo, Sri Lanka; ${ }^{3}$ National Eye Hospital, Sri Lanka; ${ }^{4}$ Department of Public Health and Family Medicine, Sir John Kotelawala Defence University, Sri Lanka

*Correspondence: anujigamage @ gmail.com

DOI: https://doi.org/10.4038/jccpsl.v26i5.8346

Received on 17 May 2020

Accepted on 25 May 2020

\section{Highlights}

- Testing provides data to inform policy decisions.

- Surveillance is the key to estimate past infection and immunity and is a guidance for policy makers in lifting preventive measures in a stepwise manner.

- Scaling up testing capacity and accessibility is essential.

- The rationale for repetitive testing on an infected person needs to be explored.

\section{Background to testing in SARS-CoV-2}

"You can't fight a virus if you don't know where it is" WHODirector General

In December 2019, a cluster of patients with pneumonia of unknown origin was reported in Wuhan, China. Subsequently, the causative pathogen was identified as Severe Acute Respiratory Syndrome-Coronavirus-2 (SARS-CoV2), which is a novel respiratory pathogen (1) comprising a singlestranded RNA that could infiltrate healthy cells to multiply and survive. The virus is isolated in several animal species (2). Since the identification of this pathogen, there has been an exponential rise in the number of cases worldwide. Several theories have been put forward to explain the rapid spread of this virus and the consequent global pandemic. These include high transmissibility of the virus (3), lack of cross protective immunity from related viral infections and delayed public health response measures (4).

To a large extent, epidemiological studies describing the disease by time, place and person have enabled the identification of effective measures against the disease (5), while testing for COVID-19 has guided disease management in individuals and shed light on 
the true rates of infection useful for making decisions on public health interventions at country level. In this backdrop, an ideal test for COVID-19 should account for several parameters: its ability to detect the infection directly (detecting the virus) or indirectly (identifying host antibodies), have an efficient test turnaround time, ability to perform tests from several patients at the same time and ability to perform in low-resource settings.

Being an infection with a high transmission rate leading to high morbidity and mortality, rapid testing is deemed necessary to contain the spread of COVID19 (2). The main testing methods used have been,

\section{- RT-PCR}

- Isothermal amplification assays

- Antigen testing

- Serology

- Medical imaging

There are two testing strategies that governments have employed, and these are testing for the presence of the active virus using swabs and testing for antibodies which indicate past infection. These two testing strategies have different uses in the response to COVID-19.

\section{- RT-PCR}

The scientists from China at a very early stage released the genome of the virus, which allowed polymerase chain reaction (PCR) tests for the virus to be developed rapidly. Direct tests for coronavirus include reverse-transcription polymerase chain reaction (RT-PCR) and real time RT-PCR (rRT PCR). However, currently only rRT-PCR is being done. These are molecular-based tests and are done to detect the current viral infection and RNA of the virus (6). In RT-PCR, the RNA virus is reversetranscribed to DNA using a specific enzyme. Then it is placed on a real time-PCR machine. In real time PCR assays, a positive reaction is detected by accumulation of a fluorescent signal. The $\mathrm{Ct}$ (cycle threshold) of PCR is defined as the number of cycles required for the fluorescent signal to achieve the threshold. Therefore, the samples with a high viral load will first get amplified resulting in a lower $\mathrm{Ct}$ value. This indicates that the viral load is inversely associated with the $\mathrm{Ct}$ value. The PCR technology is highly sensitive and specific in detecting SARSCoV-2, and therefore is considered as the gold standard. It can deliver a reliable diagnosis within three hours (6-8 hours) (7). The sensitivity of Rt-PCR rates between 71-98\% (6-7). The PCR test is done on respiratory samples obtained from the upper respiratory tract (nasopharyngeal or oropharyngeal swabs), endotracheal/ nasopharyngeal aspirates and bronchoalveolar lavage. The specimen collection should be done carefully according to the guidelines and stored at $4^{\circ} \mathrm{C}\left(2-8^{\circ} \mathrm{C}\right)$ and transported in a triple package system to the laboratory within 48 hours of collection (8). Although the rRT-PCR has high sensitivity, the accuracy would depend on the specimen collection and transportation, the site of the sample, the timing of the sample collection, handling of the sample and reagent specific issues. The analytical process too plays an essential role (2).

Limitations of the PCR method include (1) long turnaround times, a complicated operation process, (2) need for certified laboratories with expensive equipment and well-trained staff to handle samples and the machines, and (3) varying specificities for RT-PCR of COVID-19 (4, 6). Further, this test cannot detect past infections and is also limited in identifying the current viral carriage (5). Intermittent viral shedding of the virus can also contribute to varying results. In addition, the PCR test requires specialized supplies, expensive instruments and expertise of trained laboratory technicians.

\section{- Isothermal amplification assays}

Molecular testing using isothermal amplification assays provide similar sensitivities to PCR without the need for specialized laboratory equipment and requiring to be conducted only at a single temperature (5). It is a newer technique that is technically simple and easy to perform with less time, typically within 30 minutes to view results. 


\section{- Antigen testing}

Unlike the RT-PCR test which amplifies small amounts of the genetic material, there is no amplification of viral proteins in an antigen test. Certain rapid diagnostic tests (RDT) currently under development detect the presence of viral antigens expressed by the SARS-Cov-2 virus from respiratory samples. The process generates results usually within 30 minutes. However, these antigens are expressed only when there is active replication of the virus. Problems may exist with there being not enough detectable levels of antigens when using nasal swabs. According to the World Health Organization (WHO) (9), previous experience with antigen-based rapid diagnostic tests for other respiratory illnesses such as influenza has revealed test sensitivities varying from $34 \%$ to $80 \%$. While further research is encouraged on the use of these tests, the WHO currently does not recommend the use of antigen-detecting rapid diagnostic tests for patient care.

\section{- Serological tests}

Serology can be used to test for antibodies indicating past infection. Immunoglobulin M (IgM) and IgG which appear in a patient's blood can be tested for SARS-CoV-2 infection and can be regarded as a rapid, simple, and highly sensitive diagnostic test (10). It is reported that in post COVID-19 infection, IgM antibody could be detected after 3-6 days and IgG after 8 days in patient's blood (11). IgM antibodies indicate recent infection, whereas $\operatorname{IgG}$ indicates exposure to the virus some time back. The rapid antibody testing kits can detect antigens within 15 minutes and the reported sensitivity and specificity are high. However, as opposed to direct testing, this test does not indicate the presence of the virus (11) nor is it able to specify the duration of the illness. Additionally, crossreactivity with other coronaviruses and flu viruses cannot be ruled out, and therefore a negative serology test might not indicate that infection has not happened (12). However, the RT-PCR and IgM and IgG tests can be combined to provide a more accurate diagnosis of SARSCoV2 infection (10). This could be useful to assess the prevalence of asymptomatic infection amongst people and to guide COVID-19 prevention methods.
Validation of serological tests is needed to determine the diagnostic accuracy and reliability (9). The WHO does not recommend use of any rapid antibody detection kit nor antibody tests for diagnostic purposes; however, the WHO has recommended the use of $\operatorname{IgG}$ for sero-surveillance in community screening (13).

\section{- Medical imaging}

During a period of shortage of testing kits and false negative rate of rRT-PCR, China used computer tomography (CT) scans to clinically diagnose COVID-19 (14). However, the imaging features of COVID-19 are diverse and usually depend on the stage of infection identified following the onset of symptoms. Although nonspecific bilateral pneumonia is more frequently reported on chest radiography, CT is more sensitive than plain radiographs with hallmark features of COVID-19 commonly seen such as bilateral and peripheral ground-glass opacities and consolidations of the lungs. According to a study by Bernheim et al. (15), normal CT findings were frequently observed (56\%) in the early stages of the disease (0-2 days) with increasing lung involvement within 10 days after the onset of symptoms. Pan et al. (16) also found that in addition to ground glass opacities, crazy-paving patterns develop with increasing consolidation of the lungs. While studies have shown CT scans to have higher sensitivity (86-98\%) and lower false negative rates in comparison to RT-PCR, its specificity is still low as tomography findings frequently overlap with other viral pneumonias (14). It is currently recommended that $\mathrm{CT}$ findings in such patients be correlated with clinical and laboratory evidence of COVID-19 infection.

\section{Approaches used for testing}

Policies on testing differ from region to region, country to country and even from city to city. There are different approaches being adopted for testing, depending on the supplies, shortages and priorities. The following are predominantly considered when deciding on the best strategy for testing for a particular region. 
- The state of the epidemic in the country

- Availability of testing resources (test kits, laboratory facilities, personal protective equipment, human resources)

The WHO has suggested the following protocol on strategies used for laboratory testing in relation to each transmission scenario.

- When there are no cases in the community, it is recommended to test all individuals meeting the suspected case definition, to test a subset of samples from severe acute respiratory illness (SARI)/ influenza-like-illness (ILI) surveillance for COVID-19 or to test patients with unexpected clinical presentation (17).

- When there are sporadic cases or clusters of cases in a community, it is recommended to test all individuals with suspected case definition, testing of the cases and clusters of COVID-19, SARI/ILI surveillance for COVID-19.

- When there is community transmission (resource poor setting), testing is recommended for the people who are at risk of developing severe disease and for the vulnerable populations who will require hospitalization or advanced care for COVID-19. Testing should also be done on healthcare workers regardless of whether they have encountered a diagnosed case or not. In such areas, it is also recommended to test the initial symptomatic individuals in closed settings (schools, prisons, camps, etc.) for COVID-19 and all other symptomatic cases must be considered as 'probable cases'. Any suspected patient requiring admission to a healthcare facility should be tested for COVID-19. Also, any symptomatic healthcare worker with a contact history or any healthcare worker from an area where community transmission has started should be tested to ensure protection of the vulnerable patients from nosocomial infection.
- If there is an increased number of suspected cases in a specific demographic group, the WHO recommends testing a subset of these cases to decide on their diseased status. Nonetheless, since a country can simultaneously have areas with cases and areas with no cases, it would be advisable to carry out different testing strategies within the same country. For example, in areas within the country with no cases, it is advisable to test all suspected cases to find the initial case and intervene early to stop the spread. Testing in areas with community transmission should be different from the rest and prioritized to protect the vulnerable population and healthcare workers from getting infected. Testing among vulnerable populations is important to prevent progression towards more severe disease to keep the number of serious patients below the intensive care unit (ICU) capacity.

- A recovering patient who has tested negative twice can be discharged after 14 days of selfquarantine (17).

\section{Country experiences on application of the diagnostic test}

In China, which was the initial epicentre of COVID19, RT-PCR, gene sequencing and COVID-19 specific $\operatorname{IgM}$ and $\operatorname{IgG}$ antibodies were the most used diagnostic investigations. South Korea, Singapore and Hong Kong are identified as countries which contained the COVID-19 pandemic well through wide-scale testing.

South Korea adopted an aggressive strategy of testing people for coronavirus broadly, testing more than 440,000 people while adhering to other mitigation measures to confine COVID-19 (2). Also, South Korea along with Vietnam have been conducting mass scale testing. In these countries, they have fewer issues with the supply of test kits and PPE shortages. Their objective is to identify as many people as possible, including the asymptomatic patients. They also isolate the positives and trace the contacts and quarantine to minimize the spread of the infection. By following this protocol, despite being in the borders 
of China, Vietnam succeeded in controlling the spread of the disease.

As for Singapore, it increased their capacity to conduct testing for COVID-19. On average, they have been conducting 2,900 tests per day and increased it further to 8,000 per day by late April. Singapore testing strategy is based on the following considerations.

- Testing for diagnosis and surveillance - testing all symptomatic patients and for active case finding and for surveillance purposes

- Testing in the migrant worker dormitories

- Testing for protection and monitoring (i.e. to protect the vulnerable groups such as staff at nursing homes who are in close contact with seniors, healthcare workers and frontline officers who have regular contact with confirmed cases) (3).

In contrast, in the United Kingdom, patients in hospital ICUs with suspected COVID-19 have been prioritised, followed by people with severe respiratory illnesses such as pneumonia. The next priority has been isolated cluster outbreaks, such as in care homes and healthcare workers.

\section{Cost-effective strategies used for sample collection}

There is much to be determined regarding the diagnostic strategies in COVID-19. Evidence on the cost effectiveness analysis related to diagnostic methods has not yet been published to be considered as a measure for scaling-up testing and surveillance. Most governments deploy the strategy of rRT-PCR and the preferred sample for testing has been the nasopharyngeal/oropharyngeal swab. In order to improve the cost effectiveness of testing, a few countries like South Korea and USA are using drivethrough testing laboratories where nasopharyngeal swabs are being taken while the patient is in the car and the vial is directly sent for testing. The vial is then transported to the central laboratory for testing. This would save a lot of resources including time, human resource and personal protective equipment (PPEs).

Some countries like South Korea and Germany are doing aggressive testing on a mass scale. Pooled sample PCR analysis would save substantial resources for COVID-19 testing, which. can be an option for low to middle income countries. This involves testing in batches. When pooled samples test positive, individual samples are assessed. Thus, the number of tests that must be carried out is less than when individual samples are tested. This is now being carried out in India using RT-PCR technology. In the large pooled samples, borderline positive single samples might not be detected. Therefore, ideally a pool of 4-30 samples can be recommended (18).

The decision on the number of testing that a country should do must be based on resource availability and scarcity, the opportunity cost of each decision, and the consequences of trade-offs. Such analysis is essential in ensuring the best use of scarce resources and gains in efficiency (19).

\section{Public health rationale for testing}

\section{- Testing during the escalating phase of the COVID-19 pandemic}

The WHO (17) recommends active case finding and testing including contact tracing in all countries, together with monitoring of trends in COVID-19 disease at national and regional levels. It should be noted that the pandemic cannot be won without aggressive testing, therefore preparedness and readiness should include the establishment of COVID-19 testing capacity in the country and logistics need to be arranged to increase testing capacity so that each and every suspected case can undergo testing and repeated testing if the need arises.

Each country should assess its risk and rapidly implement the necessary measures at the appropriate scale to reduce both COVID-19 transmission to minimize economic, public and social impacts. A country can simultaneously have areas with no cases, clusters of cases and areas with community 
transmission. Thus, different testing strategies might be needed within the same country. Thus, collection of epidemiological information is essential to conduct risk assessments, guide preparedness and to plan response measures at national and regional levels.

Healthcare workers and uninfected vulnerable patients should be protected from nosocomial COVID-19 infection. Focused testing in healthcare facilities ensures that infection prevention and control measures can be correctly implemented, while testing among vulnerable populations and risk groups will be important for early detection, treatment and to break the chain of transmission (17).

To monitor circulation of the virus in the general population, the WHO $(13,17)$ recommends implementing testing for COVID-19 via existing national sentinel surveillance sites for ILI and SARI.

\section{- Surveillance during de-escalation of mitigation measures}

While case finding, contact tracing and quarantine needs to be followed, many countries are considering lifting of mitigation measures and phasing out 'stayat-home' policies. However, lifting measures too quickly, without appropriate monitoring and health system capacity in place, may cause a sudden resurgence of sustained community transmission (20).

De-escalation of mitigation strategies can be considered once the intensity, severity and the impact indicators on healthcare system can justify the conclusion that the incidence of COVID-19 has reduced to a level where systematic case finding and isolation, contact tracing and quarantine are sufficient to control, and ultimately contain, the pandemic within a country (21).

If de-escalation leads to an increase of COVID-19 cases, re-escalation of strict mitigation measures, surveillance and monitoring needs will need to continue as before. If de-escalation results in continued absence of COVID-19 cases, robust surveillance systems will be needed to detect community transmission.

Contact tracing, testing and quarantine will be crucial during the de-escalation phase to ensure containment, followed by enhanced sentinel ILI/SARI surveillance at regional and national level at sentinel sites, while increasing the numbers of ILI/SARI cases tested for COVID-19. Valuable information can be gained through prevalence studies using RT-PCR on the extent of community transmission while population based sero-prevalence studies could provide supplementary information regarding age-specific population immunity against COVID-19 during the different phases of the pandemic (20-21).

\section{Gaps in the existing knowledge $\&$ future research}

Rapid spread of the virus which resulted in a global pandemic is not yet fully understood even though several theories are being currently evaluated. Thus, collating evidence on grey areas related to the pandemic is of paramount importance as an attempt to control it and to identify its extent (21). In this regard, COVID-19 testing serves as an important tool to minimise the spread and impact of the virus. Testing allows identifying the infected individuals and treating them as well as isolation of those infected and tracing and quarantining of their contacts. Further, testing will inform the risk of the infection in different populations. Such knowledge is important for a country to decide on the allocation of required medical and other resources more efficiently. Yet, there are many gaps in knowledge on proper and ideal diagnostic and screening testing of COVID-19.

The place for mass scale community transmission for monitoring the progression of the epidemic as opposed to contact tracing is not yet conclusive. Also, the rationale for consecutive testing until two negative results are received by a patient to be discharged should be further explored, as countries like New Zealand perform the test only once. Allocating the limited resources on repeat testing needs to be justified using scientific evidence if dual testing is to be continued in future.

The current knowledge on COVID-19 testing could be greatly improved if all the countries are able to 
report on real time data. However, for many countries, the available data on testing are either incomplete or not available. This makes it impossible for the researchers to assess the extent and significance of their testing efforts. The testing capacity, strategy and cost-effectiveness should be made available.

Coverage of testing varies enormously across different countries. In Iceland there have been more than 100 tests for 1000 people, whereas in Indonesia only 01 test per 1000 population has been conducted (22). However, evidence on the efficiency and effectiveness of the said strategies are not proven and need to be explored.

At present, case confirmation is generally based on a positive result from a PCR test in line with the WHO recommendations, while diagnostic tests beyond PCR have not been explored or widely used $(9,17)$. Knowledge on the performance and frequency of running these tests is very limited. Consequently, the comparability across countries has been lost in addition to the difficulty of identifying the best practices related to testing and interpretation of results. It would also hinder the technical advancements and evaluating the cost effectiveness of such screening especially in poor-resourced countries.

\section{Author Declaration}

Acknowledgements: We wish to acknowledge the technical guidance provided by Dr Ishara Premathilaka, Senior Lecturer, Faculty of Medicine, University of Colombo.

Author contributions: All authors were involved in the planning of the article and in literature search. MW and AUG compiled and edited the manuscript. All authors approved the final manuscript.

\section{References}

1. Marco Cascella MR, Arturo Cuomo, Scott C. Dulebohn, Raffaela Di Napoli. Features, evaluation and treatment coronavirus (COVID-19). Treasure Island: StatPearls Publishing, 2020. Available from:https:// www.ncbi. nlm.nih.gov/ books/NBK554776/ . Accessed 15 May 2020.1

2. Zhai P, Ding Y, Wu X, Long J, Zhong Y, Li Y. The Epidemiology, diagnosis and treatment of COVID19. International Journal of Antimicrobial Agents 2020; 105955-105955. DOI: 10.1016/j.ijantimicag. 2020.105955.

3. Li Q, Guan X, Wu P, Wang X, Zhou L, Tong Y, Ren R, Leung KSM, Lau EHY, Wong JY, Xing X, Xiang N, Wu Y, Li C, Chen Q, Li D, Liu T, Zhao J, Liu M, Tu W, Chen C, Jin L, Yang R, Wang Q, Zhou S, Wang R, Liu H, Luo Y, Liu Y, Shao G, Li H, Tao Z, Yang Y, Deng Z, Liu B, Ma Z, Zhang Y, Shi G, Lam TTY, Wu JT, Gao GF, Cowling BJ, Yang B, Leung GM, Feng Z. Early transmission dynamics in Wuhan, China of novel coronavirus-infected pneumonia. New England Journal of Medicine 2020; 382(13): 1199-1207. DOI: 10.1056/ NEJMoa2001316.

4. Lee VJ, Chiew CJ, Khong WX. Interrupting transmission of COVID-19: lessons from containment efforts in Singapore. Journal of Travel Medicine 2020; 27(3): 1-5. DOI: 10.1093/jtm/ taaa039.

5. Udugama B, Kadhiresan P, Kozlowski HN, Malekjahani A, Osborne M, Li VYC, Chen H, Mubareka S, Gubbay JB, Chan WCW. Diagnosing COVID-19: the disease and tools for detection. ACS Nano 2020; 14(4): 3822-3835. DOI: 10.1093/ $\mathrm{jtm} / \mathrm{taaa} 039$.

6. Jawerth, N. How is the COVID-19 virus detected using real time RT-PCR? 2020; Available from: https://www.iaea. org/ news center/news/how-isthe-covid-19-virus-detected-using-real-time-rtpcr. Accessed 15 May 2020.

7. Okba NMA, Muller MA, Li W, Wang C, GeurtsvanKessel CH, Corman VM, Lamers MM, Sikkema RS, de Bruin E, Chandler FD, Yazdanpanah Y, Le Hingrat Q, Descamps D, Houhou-Fidouh N, Reusken CBEM, Bosch B-J, Drosten C, Koopmans MPG, Haagmans BL. SARS-CoV-2 specific antibody responses in COVID-19 patients. medRxiv 2020. DOI: 10.1101/2020.03.18.20038059.

8. Ceylon College of Physicians and Epidemiology Unit. Provisional Clinical Practice Guidelines on COVID-19 Suspected and Confirmed Patients. Ministry of Health - Sri Lanka: Sri Lanka 2020. Available from: http://www. epid.gov.lk/web/ 
images/pdf/Circulars/Corona_virus/covid19\%20cpg \%20_\%20version \%204.pdf.. Accessed 15 April 2020.

9. WHO. 2020. Advice on the use of point-of-care immunodiagnostic tests for COVID-19. Available from: https://www.who.int/news-room/ commentaries/detail/advice-on-the-use-of-pointof-care-immunodiagnostic-tests-for-covid-19. Accessed 24 April 2020.

10. Li Z, Yi Y, Luo X, Xiong N, Liu Y, Li S, Sun R, Wang Y, Hu B, Chen W, Zhang Y, Wang J, Huang B, Lin Y, Yang J, Cai W, Wang X, Cheng J, Chen Z, Sun K, Pan W, Zhan Z, Chen L, Ye F. Development and clinical application of a rapid $\operatorname{IgM}-\operatorname{IgG}$ combined antibody test for SARS-CoV-2 infection diagnosis. Journal of Medical Virology 27 February 2020: 1-7. DOI: 10.1002/jmv.25727.

11. Hoffman T, Nissen K, Krambrich J, Rönnberg B, Akaberi D, Esmaeilzadeh M, Salaneck E, Lindahl J, Lundkvist Å. Evaluation of a COVID-19 IgM and IgG rapid test; an efficient tool for assessment of past exposure to SARS-CoV-2. Infection Ecology \& Epidemiology 2020; 10(1): 1754538. DOI: 10.1080/20008686.2020.1754538.

12. CDC. 2020. Interim Guidelines for COVID-19 Antibody Testing: Interim Guidelines for COVID19 Antibody Testing in Clinical and Public Health Settings. Laboratories. Available from: https://www.cdc.gov/corona virus/2019. ncov/lab/resources/antibody-tests-guidelines. html. Accessed 31 March 2020.

13. WHO. 2020. Surveillance strategies for COVID-19 human infection. Available from: https://www. who.int/publications/i/item/surveillance-strategiesfor-covid-19-human-infection. Accessed 15 May 20.

14. Ai T, Yang Z, Hou H, Zhan C, Chen C, Lv W, Tao Q, Sun Z, Xia L. Correlation of chest CT and RT-PCR testing in coronavirus disease 2019 (COVID-19) in China: a report of 1014 cases. Radiology 2020; 200642. DOI: 10.1148/radiol.2020200642.

15. Bernheim A, Mei X, Huang M, Yang Y, Fayad ZA, Zhang N, Diao K, Lin B, Zhu X, Li K, Li S, Shan H, Jacobi A, Chung M. Chest CT findings in coronavirus disease-19 (COVID-19): relationship to duration of infection. Radiology 2020; 200463. DOI: 10.1148/radiol. 2020200463.

16. Pan F, Ye T, Sun P, Gui S, Liang B, Li L, Zheng D, Wang J, Hesketh RL, Yang L, Zheng C. Time course of lung changes on chest ct during recovery from 2019 novel coronavirus (COVID-19) pneumonia. Radiology 2020; 200370. DOI: 10.1148/radiol.2020200370.

17. WHO. Laboratory testing strategy recommendations for COVID-19. Available: https://apps.who.int/iris/bitstream/handle/10665/3 31509/WHO-COVID-19-lab_testing-2020.1eng.pdf. Accessed 20 April 2020.

18. Lohse S, Pfuhl T, Berkó-Göttel B, Rissland J, Geißler T, Gärtner B, Becker SL, Schneitler S, Smola S. Pooling of samples for testing for SARSCoV-2 in asymptomatic people. The Lancet Infectious Diseases 2020. DOI: 10.1016/S14733099(20)30362-5.

19. Donaldson C, Mitton C. Coronavirus: where has all the health economics gone? International Journal of Health Policy and Management 20201-3. DOI: 10.34172/ijhpm.2020.108.

20. Gilbert M, Dewatripont M, Muraille E, Platteau J-P, Goldman M. Preparing for a responsible lockdown exit strategy. Nature Medicine 2020; 26(5): 643644. DOI: 10.1038/s41591-020-0871-y.

21. ECDC, Strategies for the surveillance of COVID-19 2020. Available from https://www.ecdc. europa.eu/sites/default/files/documents/COVID19-surveillance-strategy-9-Apr-2020.pdf. Accessed 13 April 2020.

22. Hellewell J, Abbott S, Gimma A, Bosse NI, Jarvis CI, Russell TW, Munday JD, Kucharski AJ, Edmunds WJ, Sun F, Flasche S, Quilty BJ, Davies N, Liu Y, Clifford S, Klepac P, Jit M, Diamond C, Gibbs H, van Zandvoort K, Funk S, Eggo RM. Feasibility of controlling COVID-19 outbreaks by isolation of cases and contacts. The Lancet Global Health 2020; 8(4): e488-e496. DOI: 10.1016/S2214-109X(20)30074-7. 